\title{
Electronic structure and optical properties of $\mathrm{TaC}$ from the first principles calculation
}

\author{
M. Sahnoun ${ }^{1, a}$, C. Daul ${ }^{1}$, J.C. Parlebas ${ }^{2}$, C. Demangeat ${ }^{2}$, and M. Driz ${ }^{3}$ \\ 1 Département de Chimie, Université de Fribourg, Pérolles, 1700 Fribourg, Switzerland \\ 2 IPCMS-GEMM, UMR 7504 CNRS, 23, rue du Loess, 67034 Strasbourg, Cedex 2, France \\ 3 Laboratoire de Sciences des Matériaux, Université Djillali Liabes, DZ-22000 Sidi Bel Abbes, Algeria
}

Received 10 January 2005

Published online 28 April 2005 - (C) EDP Sciences, Società Italiana di Fisica, Springer-Verlag 2005

\begin{abstract}
The electronic and optical properties of tantalum carbide TaC have been calculated using the full-potential linearized augmented-plane-wave method within the local density approximation scheme for the exchange-correlation potential. We find that the optical spectra can be extremely sensitive to the Brillouin zone sampling. The influence of relativistic effects on the dielectric function is investigated. It is shown that the scalar-relativistic correction is much more important than spin-orbit coupling. Our results are found to be in good agreement with the available experimental data. The determinant role of a band structure computation with respect to the analysis of optical properties is discussed.
\end{abstract}

PACS. 71.15.Ap Basis sets (LCAO, plane-wave, APW, etc.) and related methodology (scattering methods, ASA, linearized methods, etc.). - 74.25.Jb Electronic structure. - 74.25.Gz Optical properties.

\section{Introduction}

Tantalum carbides are refractory materials widely employed as coatings in the field of high temperature technology because of their peculiar physical and chemical properties ranging from high thermal stability to good resistance against corrosion $[1-5]$. TaC is usually a non-stoichiometric material $\mathrm{TaC}_{x}$ with $0 \lesssim x \lesssim 1$, it presents a rock-salt structure and has a melting point of about $3880{ }^{\circ} \mathrm{C}$, a Knoop hardness of $2000 \mathrm{~kg} / \mathrm{mm}^{2}$ [4] and a superconducting transition temperature of $\sim 10 \mathrm{~K}$. The material has attracted much interest, but good understanding has been elusive because, as in other transition metal carbides and nitrides [5-12], TaC exists over a wide range of compositions outside stoichiometry and its physical properties vary as a consequence. Mechanical, thermal, electrical and optical properties have been investigated from both theoretical and experimental points of view [13-17]. However, to our knowledge, up to now, there has been no calculation of the optical properties of tantalum carbide that includes the full transition matrix. The determination of optical properties requires, apart from the Kohn-Sham eigenvalues, the explicit use of the wave functions which should be calculated as accurately as possible.

The aim of this paper is to present the results of a theoretical investigation of the electronic structure and optical

\footnotetext{
a e-mail: mohammed.sahnoun@unifr.ch
}

spectra of a bulk $\mathrm{TaC}$ which crystallizes in a NaCl-type lattice. The availability of ellipsometry measurements data from Modine et al. [18] was a further motivation. This kind of data is usually interpreted using the optical spectra estimated from the joint density of states [19]. The method adopted in the present work for the calculation of the optical properties of $\mathrm{TaC}$ is the full potential linearized augmented plane wave (FP-LAPW) method [20-25].

The organization of the paper is as follows: we explain the computational method in Section 2. The results are presented and discussed in Section 3 for the electronic structure and the optical properties. A brief conclusion is drawn in Section 4.

\section{Computational method}

We have carried out self-consistent calculations for $\mathrm{TaC}$ in rock-salt structure, using the FP-LAPW method in a recent implementation [22-25]. This approach has been extensively tested and is among the most accurate methods for performing electronic structure calculations of crystals. In this calculational scheme, there are no shape approximations to the charge density or potential. The exchange and correlation potential within the Local Density Approximation (LDA) is calculated using the scheme of Hedin-Lundqvist [26]. In the FP-LAPW method, the unit cell is divided into two parts: (i) non-overlapping atomic spheres (centered at atomic sites) and (ii) an interstitial 
region. The sphere radii used in the calculations for Ta and $\mathrm{C}$ are 2.1 and 1.9 a.u. respectively. Within these spheres, the potential is expanded as

$$
V(r)=\sum_{l m} V_{l m}(r) Y_{l m}(\hat{r})
$$

where $V_{l m}(r)$ are the radial functions and $Y_{l m}(\hat{r})$ the spherical harmonics. And outside the sphere

$$
V(r)=\sum_{K} V_{K} e^{i K r}
$$

where $K$ are the reciprocal lattice vectors. The Brillouin zone integrations have been carried out using $35 k$-points in the corresponding irreducible wedge. Well converged solutions are obtained for $R_{M T} \mathrm{~K}_{\max }=9, R_{M T}$ labelling the atomic sphere radii and $K_{\max }$, the interstitial plane wave cut-off.

For the calculation of the optical properties, the Brillouin zone integration is performed using the tetrahedron method [27] for a series of $k$ point sampling. The complex dielectric function:

$$
\varepsilon(\omega)=\varepsilon_{1}(\omega)+i \varepsilon_{2}(\omega)
$$

is known to describe the optical response of the medium at all photon energies. In metals, there are two contributions to intraband and interband transitions. The interband transitions can be further split into direct and indirect transitions. Both the intraband transitions and the indirect interband transitions must involve a third particle (e.g., a phonon or an impurity; see for example reference [28]) in addition to the electrons and photons in order to account for the momentum transfer. These transitions are thus three-particle interactions, which considerably complicate a calculation from first principles. However, the intraband part of the dielectric function can be modelled by using Drude's formula [29].

$$
\begin{gathered}
\varepsilon_{2}^{i n t r a}(\omega)=\frac{\Gamma \omega_{p}^{2}}{\omega\left(\omega^{2}+\Gamma^{2}\right)} \\
\varepsilon_{1}^{i n t r a}(\omega)=1-\frac{\omega_{p}^{2}}{\left(\omega^{2}+\Gamma^{2}\right)} \\
\omega_{p}^{2}=\frac{\hbar^{2} e^{2}}{\pi m^{2}} \sum_{n} \int_{k}\left|p_{n, n, k}\right|^{2} \delta\left(E_{n, k}-E_{F}\right)
\end{gathered}
$$

where $\omega_{p}$ is the free electron plasma frequency, $\Gamma$ is a lifetime broadening, and $p_{n, n, k}$ is the momentum matrix element evaluated for the LAPW basis set. The intraband transitions dominate at low energies. The indirect interband transitions are, at the current state of theoretical development, not possible to calculate explicitly. However, we can expect them to add a rather smooth background to the spectra [30]. The direct interband contribution to the imaginary part of the dielectric function, $\varepsilon_{2}^{\text {inter }}$, is calculated by summing all possible transitions from occupied to unoccupied states, taking the appropriate transition matrix element into account [31]. The real part $\varepsilon_{1}^{\text {inter }}$ is then determined from $\varepsilon_{2}^{\text {inter }}$ by imposing the condition of causality [32]. All optical constants may now be derived from the preceding considerations. Let us just recall the main expressions. The imaginary part of the dielectric function is given as usual [33]:

$$
\begin{gathered}
\varepsilon_{2}(\omega)=\left(\frac{\hbar^{2} e^{2}}{\pi m^{2} \omega^{2}}\right) \sum_{i, j} \int_{k}\left|p_{i, j, k}\right|^{2}\left(f\left(E_{i, k}\right)-f\left(E_{j, k}\right)\right) \\
\times \delta\left(E_{j, k}-E_{i, k}-\omega\right) d^{3} k
\end{gathered}
$$

where $i$ and $j$ are the initial and final states, respectively, $f$ is the Fermi distribution function, and $E_{i}$ is the energy of an electron in the $i$ th state. The real part $\varepsilon_{1}(\omega)$ of the dielectric function can be extracted from the imaginary part using the Kramers-Kronig relation:

$$
\varepsilon_{1}(\omega)=1+\frac{2}{\pi} P \int_{0}^{\infty} \frac{\omega^{\prime} \varepsilon_{2}\left(\omega^{\prime}\right) d \omega^{\prime}}{\left(\omega^{\prime 2}-\omega^{2}\right)}
$$

where $P$ is the principal value of the integral.

The optical spectra such as the refractive index $n(\omega)$, the extinction coefficient $k(\omega)$ and the absorption coefficient $\alpha(\omega)$ are easily calculated in terms of the components of the complex dielectric function, i.e.:

$$
\begin{gathered}
n(\omega)=\left[\frac{\varepsilon_{1}(\omega)}{2}+\frac{\sqrt{\varepsilon_{1}(\omega)^{2}+\varepsilon_{2}(\omega)^{2}}}{2}\right]^{1 / 2} \\
k(\omega)=\left[\frac{\sqrt{\varepsilon_{1}(\omega)^{2}+\varepsilon_{2}(\omega)^{2}}}{2}-\frac{\varepsilon_{1}(\omega)}{2}\right]^{1 / 2} \\
\alpha(\omega)=\sqrt{2} \omega\left(\sqrt{\varepsilon_{1}(\omega)^{2}+\varepsilon_{2}(\omega)^{2}}-\varepsilon_{1}(\omega)\right)^{1 / 2} .
\end{gathered}
$$

\section{Results and discussion}

In order to fully interpret the optical spectra which arise from interband transitions, we briefly describe the electronic properties of TaC. Fitting the Murnaghan equation of state [34] to the total energies versus lattice volume yields the equilibrium lattice constant $\left(a_{e q}=4.42 \AA\right)$, the bulk modulus ( $\left.B_{0}=369.83 \mathrm{GPa}\right)$ and the pressure derivative of the bulk modulus $\left(B^{\prime}=4.58\right)$. When the equilibrium lattice constant is compared to the experimental value $(a=4.456 \AA)[4,35]$, we find that our calculated result is rather close to the experimental value.

In Figure 1, we present the total and projected densities of states (DOS) for TaC. The main peak above the Fermi level is located at $4 \mathrm{eV}$. Below the Fermi level, there are three main peaks, located at $-5,-7$, and $-12 \mathrm{eV}$. According to the projected DOS it is clear that the lowest energy structure is mainly composed of the $\mathrm{C} 2 s$ band with a little admixture of Ta $5 d$ states. The next two structures below the Fermi level primarily contain electrons from C $2 p$ bands and from the Ta $5 d$ bands. A broad structure at about $4 \mathrm{eV}$ above the Fermi level contains states arising from the Ta $5 d$ bands and to a lesser extend from 

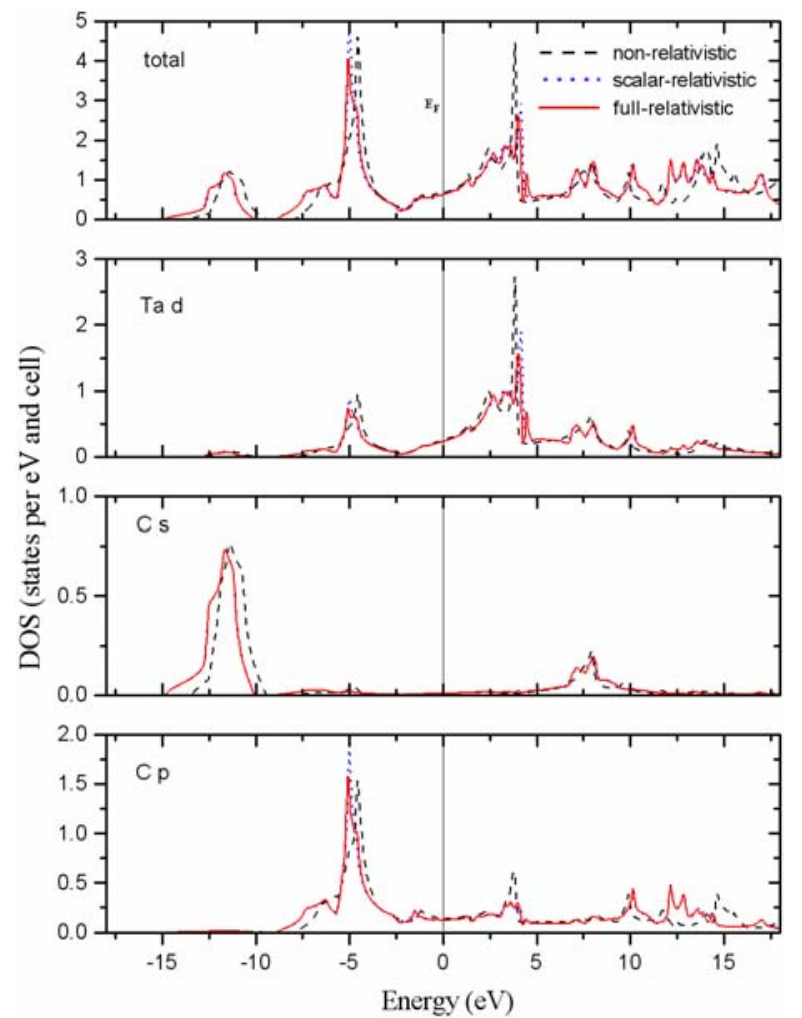

Fig. 1. Total and site-dependent densities of states of TaC from a non-relativistic (dashed line), a scalar-relativistic (dotted line) and full-relativistic calculation (full line).
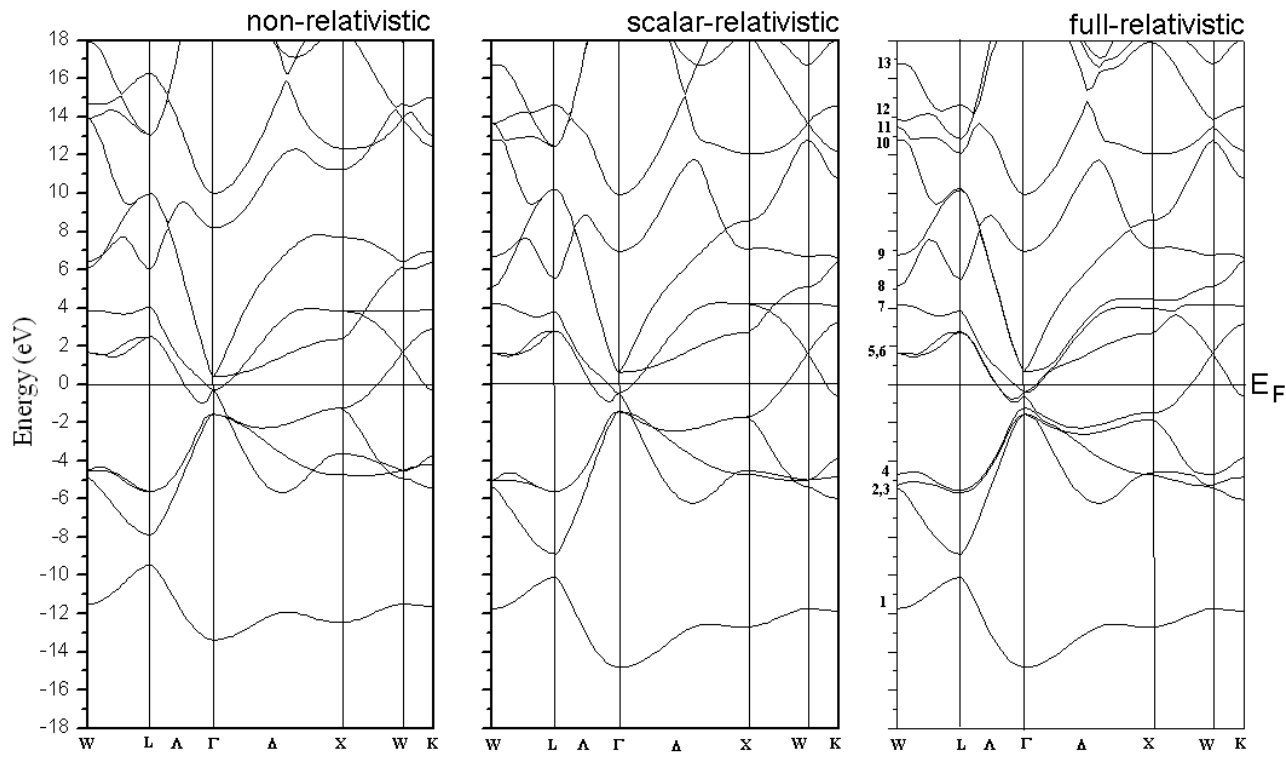

Fig. 2. Band structure of TaC: non-relativistic (left), scalar-relativistic (middle) and full-relativistic (right) have been performed.

the $\mathrm{C} 2 p$ bands. The overall density of states we obtained for $\mathrm{TaC}$ is in good overall agreement with the corresponding results from other authors [36,37]. Further support for our theoretical results stems from the good agreement with the measured peaks separations which is independent of the uncertainty in determining the position of $E_{F}$ from the experimental data.

A straightforward comparison between the theoretical density of states at the Fermi energy and the specific heat coefficient at low temperature is possible. Unfortunately, the only data available, we are aware of, is the specific heat of $\mathrm{TaC}$ at $298 \mathrm{~K}$ [4] which is not directly obtained in our calculation. Nevertheless, our prediction of 0.63 states $/ \mathrm{eV} /$ cell is in good accord with other theoretical works $[15,17,38,39]$.

The electronic band structure of $\mathrm{TaC}$ along several lines of high symmetries is also shown in Figure 2 in order to further illustrate our optical results. Let us just 


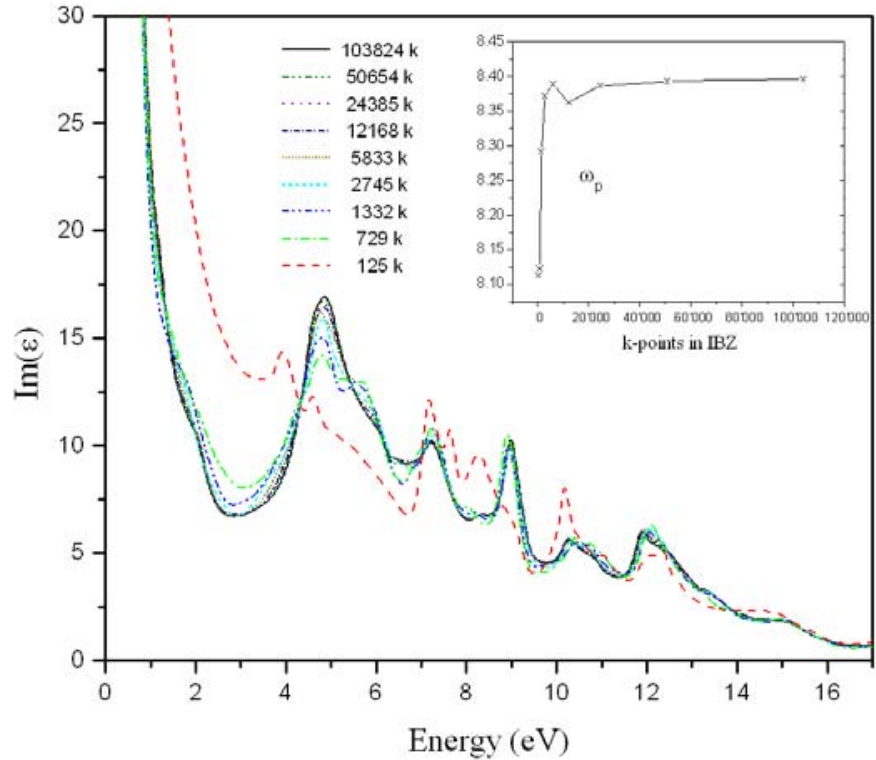

Fig. 3. Imaginary part of the frequency dependent dielectric function of $\mathrm{TaC}$ for a series of $k$ point numbers in the IBZ. In the inset the dependence of the plasma frequency (in $\mathrm{eV}$ ) with respect to the $k$ point numbers.

give a brief description of a few band dispersions. Band 1 has been indexed starting from the lowest energy: it arises from the C $2 s$ state and does not contribute to the bonding. It displays a maximum dispersion at the $L$ point at an energy of $-10.45 \mathrm{eV}$. The next lowest bands originate mainly from C $2 p$ states $\left(\Gamma_{15}\right.$, at $\left.\Gamma\right)$ and Ta $5 d$ states $\left(\Gamma_{12}\right.$ and $\Gamma_{25^{\prime}}$, at $\Gamma$ ). Actually, the Ta $5 d$ bands are further decomposed into $\mathrm{t}_{2 g}$ originating from $\Gamma_{25^{\prime}}$ and $\mathrm{e}_{g}$ originating from $\Gamma_{12}$. The highest band shown in Figure 2 originates from the $\mathrm{Ta} 6 s$ state because of a repulsive interaction with the previously considered C $2 s$ band which shifts the Ta $6 s$ states to higher energies above the Fermi level. The Ta $6 s$ states do not mix very much with the Ta $5 d$ states, but rather do lie at about $5.8 \mathrm{eV}$ above the $\Gamma_{12}$ level, at $\Gamma$ for example.

In terms of convergence with respect to the most important parameters, our results did not turn out to be sensitive to the number of LAPW's, but there is a crucial dependence upon the BZ sampling.

Figure 3 shows the interband contribution to the imaginary part of the dielectric function, obtained in a scalarrelativistic calculation, for a series of $k$ point meshes. The peaks do not only get sharper with increasing the number of $k$ points, but they also exhibit a pronounced energy shift. Only with the most dense mesh, quantitative agreement with experimental data is achieved (Fig. 4) [18]. The inset of Figure 3 exhibits the plasma frequency as a function of the number of $k$ points in the irreducible wedge of the Brillouin zone (IBZ). We note that the plasma energy of the full-relativistic calculation changes by $0.27 \mathrm{eV}$, i.e. from $8.11 \mathrm{eV}$ obtained with $125 k$-points to the converged value of $8.38 \mathrm{eV}$ obtained with about $1000 k$-points or more. Since the main contributions stem from certain weighted regions of the BZ, a refinement of the mesh can

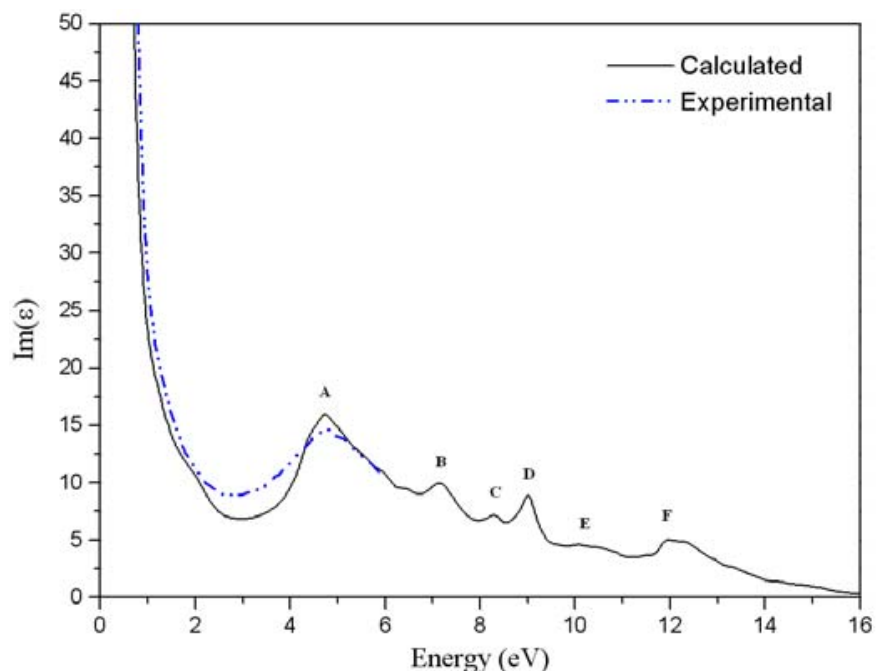

Fig. 4. Calculated scalar-relativistic imaginary part of the dielectric function including a life-time broadening of $0.1 \mathrm{eV}$ (full line), compared (up to $6 \mathrm{eV}$ ) to the corresponding experimental data [18] (dotted-dashed line).

significantly add new contributions to the total spectrum. The experimental measurements yield the plasma energy values at 18, 7.9 and $9 \mathrm{eV}$, as estimated from reflectance data, phase data and carrier concentration studies, respectively [18]. Our calculated value of $8.38 \mathrm{eV}$ is in reasonable agreement with both values obtained by fitting phase data and carrier concentrations, but the value obtained from the reflectance data appear to be too large.

In order to investigate relativistic effects we have performed three types of calculations: non-relativistic, scalarrelativistic, and including spin-orbit coupling up to second order. In Figure 5, both the real and the imaginary parts of the energy dependent dielectric function are shown for the three cases mentioned above. While there is less difference between the scalar-relativistic and the one including spin-orbit, the change is more substantial when going from non-relativistic to scalar-relativistic approximations. Let us just recall here that the scalar-relativistic effects include both Darwin shift, and mass-velocity corrections.

In Figure 4, we present the imaginary part of the dielectric function for $\mathrm{TaC}$ including a life-time broadening of $0.1 \mathrm{eV}$ (which also partially expresses the experiments resolution) in order to better compare to the corresponding data given by Modine et al. [18]. Actually we obtain a reasonable agreement below $6 \mathrm{eV}$ where the comparison with experiment is available. At low energy, where intraband transitions occur, an abrupt rise shows up below $1 \mathrm{eV}$, and a minimum at about $3 \mathrm{eV}$, which confirms the low energy divergence seen in metallic materials and the overall spectral shape somewhat similar to the ones corresponding to other comparable transition metals.

At higher energies and using our calculated band structures it is worthwhile to identify the interband transitions that are responsible for the peak structures of $\varepsilon_{2}(\omega)$ labelled A, B, C, D, E and F in Figure 4 and corresponding to the following energies: $4.66 \mathrm{eV}$ (A), $7.06 \mathrm{eV}$ (B), $8.2 \mathrm{eV}(\mathrm{C}), 8.9 \mathrm{eV}(\mathrm{D}), 10.7 \mathrm{eV}(\mathrm{E})$ and $11.8 \mathrm{eV}(\mathrm{F})$. We 


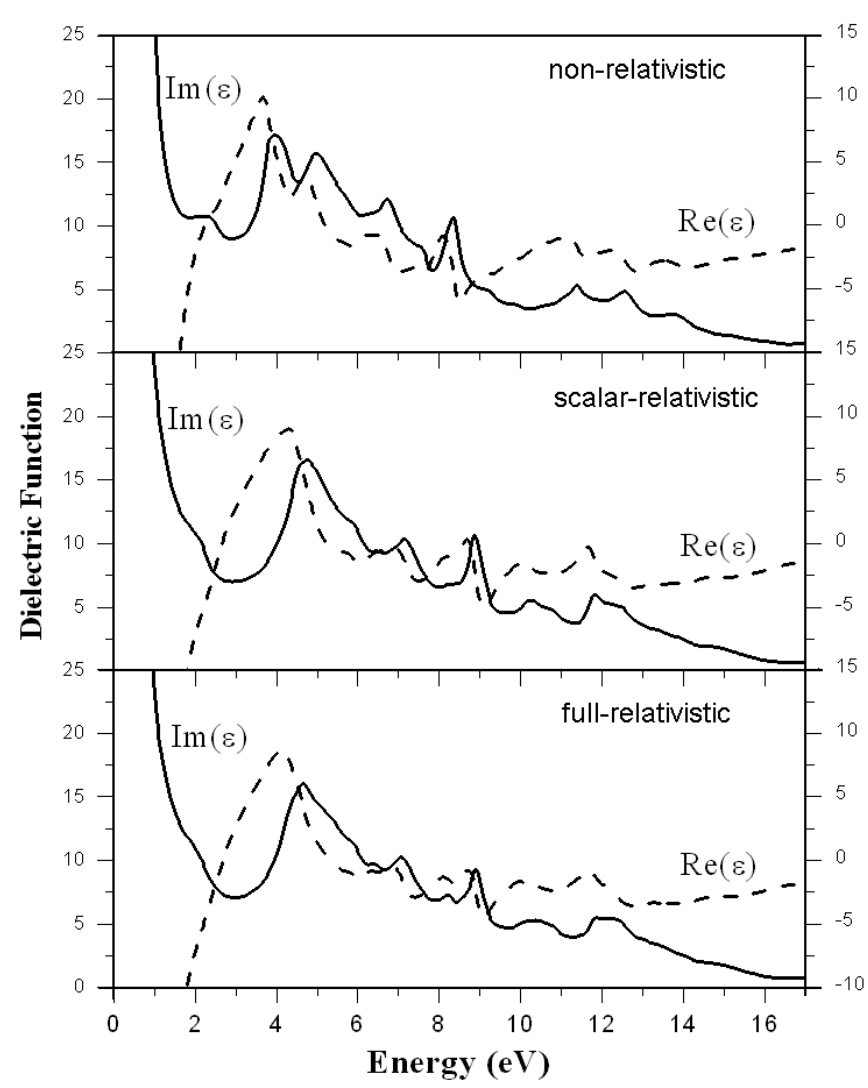

Fig. 5. Calculated energy dependent dielectric function of TaC: real (dashed lines) and imaginary part (full lines) of from non-relativistic (top), scalar-relativistic (middle) and fullrelativistic calculations (bottom).

need then to look at the optical matrix elements for the various pairs of bands which are involved to eventually obtain peaks in the imaginary part of the dielectric function. Thus, we notice that the major peak at about $4.66 \mathrm{eV}$ corresponds to the transition from the high density of states region at $-4.7 \mathrm{eV}$ (band 4) to the empty bands located above the Fermi level. The peak at $7.06 \mathrm{eV}$ can be related to transitions between bands 4 and 6 (along $\mathrm{K}$ ). The peaks at 8.9 and $11.8 \mathrm{eV}$ show the direct transitions between bands 2 and 7 (along $\mathrm{X}$ ) and between bands 4 and 9 (along $\mathrm{W}$ ), respectively.

\section{Conclusion}

We used the FP-LAPW method, within the LDA for exchange correlation potential in order to investigate the electronic structure and the optical properties of $\mathrm{TaC}$ compound. Three types of calculations were performed: non-relativistic, scalar-relativistic and full-relativistic. Using the energy band dispersion we analyzed the interband contributions to the optical properties and explained the origin of various features observed in the optical spectra, according to the predicted energy band dispersion curves. We obtained quite good agreement with experiment in terms of plasma frequency and of the position of the first interband transition peak. This agreement can, however, only be achieved when the BZ integration is carried out with sufficient accuracy (large number of $k$-points). The optical properties of $\mathrm{TaC}$ are significantly influenced by the scalar-relativistic effects and to a lesser extend by the spin-orbit coupling.

This work was supported by the Swiss National Science Foundation and the Swiss State Secretariat for Education and Science.

\section{References}

1. G. Georgeiev, N. Fescheschiev, D. Popov, Z. Uzuuov, Vacuum 36, 595 (1986)

2. P.K. Ashwini, V. Kumarand, J. Sarkar, J. Vac. Sci. Technol. A 7, 1488 (1989)

3. J. Veligdan, D. Branch, P.E. Vanier, R.E. Barletta, Mater. Res. Soc. Symp. Proc. 285, 320 (1983)

4. A. Krajewski, L. D'Alessio, G. De Maria, Cryst. Res. Technol. 33, 341 (1998)

5. R. Teghil, L. D'Alessio, M. Zaccagnino, D. Ferro, V. Marotta, G. De Maria, Appl. Surf. Sci. 173, 233 (2001)

6. A.J. Scott, R. Brydson, M. MacKenzie, A.J. Craven, Phys. Rev. B 63, 245105 (2001)

7. I. Pollini, A. Mosser, J.C. Parlebas, Phys. Rep. 355, 1 (2001)

8. H.M. Benia, M. Guemmaz, G. Schmerber, A. Mosser, J.C. Parlebas, Appl. Surf. Sci. 200, 231 (2002)

9. H.M. Benia, M. Guemmaz, G. Schmerber, A. Mosser, J.C. Parlebas, Appl. Surf. Sci. 211, 146 (2003)

10. C. Stampfl, A.J. Freeman, Phys. Rev. B 67, 064108 (2003)

11. M. Torche, G. Schmerber, M. Guemmaz, A. Mosser, J.C. Parlebas, Thin Solid Films 436/2, 208 (2003)

12. A.I. Gusev, A.A. Rempel, V.N. Lipatnikov, J. Phys.: Condens. Matter 8, 8277 (1996)

13. P. Weinberger, R. Podloucky, C.P. Mallett, A. Neckel, J. Phys. C: Sol. St. Phys. 12, 5, 801 (1979)

14. S. Thomas, E.B. Pattinson, J. Phys. D: Appl. Phys. 2, 11, 1539 (1969)

15. B.M. Klein, D.A. Papaconstantopoulos, L.L. Boyer, Phys. Rev. B 22, 1946 (1980)

16. M. Gupta, Phys. Rev. B 14, 5205 (1976)

17. H. Ihara, M. Hirabayashi, H. Nakagawa, Phys. Rev. B 14, 1707 (1976)

18. F.A. Modine, R.W. Major, T.W. Haywood, G.R. Gruzalski, D.Y. Smith, Phys. Rev. B 29, 836 (1984)

19. A.L. Ivanaovsky, V.P. Zhukov, V.A. Gubanov, Electronic Structure of Refractory Carbides and Nitrides of Transition Metals (Nauka, Moscow 1990)

20. D.J. Singh, Plane Waves, Pseudopotentials and the LAPW Method (Kluwer Academic Publishers, Boston 1994)

21. M. Weinert, E. Wimmer, A.J. Freeman, Phys. Rev. B 26, 4571 (1982)

22. P. Blaha, K. Schwarz, G.K.H. Madsen, D. Kvasnicka, J. Luitz, WIEN2k, An augmented plane wave plus local orbitals program for calculating crystal properties (Vienna University of Technology, Austria 2001)

23. P. Blaha, K. Schwarz, J. Luitz, Wien97, Vienna university of technology Improved and Updated Unix Version of the original copyrighted WIEN-code (1997) 
24. P. Blaha, K. Schwarz, P. Sorantin, S.B. Trickey, Comput. Phys. Commun. 59, 399 (1990)

25. P.I. Sorantin, Ph.D. Thesis (Technische Universität Wien, Vienna 1990)

26. L. Hedin, B.I. Lundqvist, I. Phys. C 4, 2064 (1971)

27. P.E. Blöchl, O. Jepsen, O.K. Andersen, Phys. Rev. B 49, 16223 (1994)

28. J.C. Parlebas, D.L. Mills, Phys. Rev. B 17, 3853 (1978)

29. P. Drude, Ann. Phys. (Leipzig) 1, 566 (1900); P. Drude, Ann. Phys. (Leipzig) 3, 369 (1900)

30. N.V. Smith, Phys. Rev. B 3, 1862 (1971)

31. P. Puschnig, C. Ambrosch-Draxl, Phys. Rev. B 66, 165105 (2002)
32. J.S. Toll, Phys. Rev. 104, 1760 (1956)

33. L.D. Landau, E.M. Lifshitz, Electrodynamics in Continuous Media (Pergamon, Oxford, 1960)

34. F.D. Murnaghan, Proc. Natl. Acad. Sci. USA 3, 244 (1944)

35. M. Terrones, W.K. Hsu, A. Schilder, H. Terrones, N. Grobert, J.P. Hare, Y.Q. Zhu, M. Schwoerer, K. Prassides, H.W. Kroto, D.R.M. Walton, Appl. Phys. A 66, 307 (1998) 36. E.Z. Kurmaev, A. Moewes, Z.V. Pchelkina, I.A. Nekrasov, A.A. Rempel, D.L. Ederer, Phys. Rev. B 64, 073108 (2001) 37. O.Yu. Khyzhun, J. Alloys and Compounds 259, 47 (1997) 38. H.Z. Zeller, Phys. Rev. B 5, 1813 (1972)

39. M. Gupta, A.J. Freeman, Phys. Rev. B 14, 5205 (1976) 